\title{
The effect of Omega-3 solution in the prevention of epidural fibrosis formation in experimental rabbit hemilaminotomy model
}

\author{
Cengiz Çokluk*a, Keramettin Aydın $a$, Levent Tumkaya $b$ \\ a Department of Neurosurgery, Medical Faculty, Ondokuz Mayis University, Samsun, Turkey

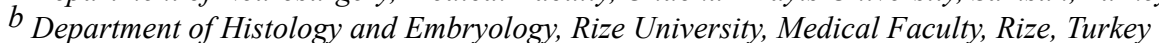

\begin{tabular}{|c|c|}
\hline ARTICLI & \\
\hline Article $\mathbf{H}$ & \\
\hline Received & 17 / 02 / 2010 \\
\hline Accepted & 05 / 03 / 2010 \\
\hline * Corresp & lce to: \\
\hline Cengiz $\mathrm{Co}$ & \\
\hline Ondokuz & University \\
\hline Medical F & \\
\hline Departme & Neurosurgery \\
\hline $55139 \mathrm{San}$ & Turkey \\
\hline e-mail: ce & kluk@yahoo.com \\
\hline
\end{tabular}

\section{Key Words :}

Peridural Fibrosis

Fatty Acids

Hemilaminotomy

Omega-3

Lumbar spine

Rabbit

\section{ABSTRACT}

The aim of this experimental study was to evaluate the effect of omega-3 fatty acids solution in the prevention of the forming of peridural fibrotic tissue and adhesion in a rabbit hemilaminotomy model. This new experimental model was previously developed by the first author of this study. Twenty-one New Zealand white rabbits were used for this experiment. Seven of them were randomly selected as Control group and was named as Group I rabbits. The rabbits in this group were not operated and treated. The second group was Group II including 7 rabbits. This group was named as hemilaminotomy and untreated rabbits. Group III included 7 rabbits and named as Omega-3 treated group after hemilaminotomy procedure. In this group (Group III), $250 \mathrm{mg}$ omega-3 solution was put in the hemilaminotomy area before closing the skin and fascia. Four weeks later from the operation rabbits were sacrificied by perfusion with $10 \%$ neutral buffered formalin solution. The lumbar spines were removed and immersed in $10 \%$ neutral buffered formalin for approximately 24 hours. Then each specimen was decalcified in 5\% formic acid for approximately 3 weeks. Specimens were cut coronally for gross inspection. Peridural scar tissue on gross inspection in coronal sectioned specimens was graded into three grades. Grade I epidural scar tissue was scored with 1 point, Grade II with 2 point and Grade III with 3 point. The average score of hemilaminotomy sites without treatment was found as $2,6 \pm 0,51$. In the other hand the average score of hemilaminotomy sites with omega-3 fatty acids treatment was found as $1,9 \pm 0,56$. The difference between the treatment and non-treatment group was statistically significant. Omega-3 fatty acids solution seems an effective treatment modality in the prevention of peridural excessive scar tissue formation in the rabbit hemilaminotomy model.

J. Exp. Clin. Med., 2010; 27:16-19

(C) 2010 OMU All rights reserved

\section{Introduction}

Peridural scar tissue formation is multifactorial in origin (Gabriel and Friedman, 1996; Dogulu et al., 2003). Many factors may take a role in the developing of this pathological process (Gabriel and Friedman, 1996; Dogulu et al., 2003). Peridural fibrosis is a cause of Failed Back Surgery Syndrome being reported as a cause of symptoms in $24 \%$ of these patients (Gabriel and Friedman, 1996).

Although many researches focused on the prevention offibrotic scar tissue which developed around the dura mater and spinal roots, this pathological process still is a cause of unsuccessful lumbar disc surgery (Robertson et al., 1993; Gabriel and Friedman, 1996; Dogulu et al., 2003). Minimally invasive surgical procedures, microsurgical techniques, and adequate hemostasis may not be sufficient to prevent epidural fibrotic tissue formation (Gabriel and Friedman, 1996; Dogulu et al., 2003).

In this experimental study, we evaluated the effect of Omega-3 fatty acids solution in the developing of peridural scar tissue formation after rabbit hemilaminotomy procedure.

\section{Materials and Methods}

Twenty New Zealand white, female rabbits weighing 250-350 gram were used for this experimental study. All rabbits were grouped into two category as Group I and II. Group I (hemilaminotomy group) was selected as control group. This group included 10 rabbits. Hemilaminotomy procedure was performed in all rabbits in this group. Group II (omega-3) was selected as Omega-3 group. This group also included 10 rabbits. $250 \mathrm{mg}$ omega-3 solution (Marincap, $500 \mathrm{mg}$ liquid omega-3 containing capsule, Kocak drug co. Istanbul) was put on the hemilaminotomy surface in all rabbits in this group.

The animals were housed in same room but seperate individual cages at the University of Ondokuz Mayıs, in 
the Animal Laboratory for Surgical Research. The animals were allowed free access to food and water in their cages with a 12 hour light/dark cycle and a temperature of $22^{\circ} \mathrm{C}$. All the rabbits were fed a standard rabbit diet and given 3-4 days for acclimatization to the new surroundings in the animal laboratory in order to reduce the stress which caused by transportation and changing of the environment.

The rabbits were anesthetized with Ketamine (35 $\mathrm{mg} / \mathrm{kg})$ and Xylazine $(5 \mathrm{mg} / \mathrm{kg})$. The lower half of the back was shaved with an electric clipper. A preoperative dose of intramuscular penicillin $(30000 \mathrm{U} / \mathrm{kg})$ was given. Each of the rabbits was positioned prone on the operating table above a heating blanket with slight lumbar flexion produced by a small towel placed beneath its abdomen. A mixture of oxygen with nitrous oxide and $1 \%$ to $2 \%$ halothane was administered via a face mask to provide anesthesia and analgesia.

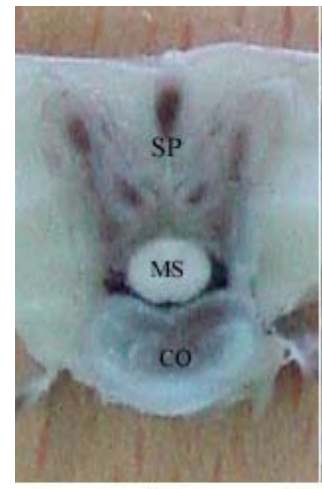

A

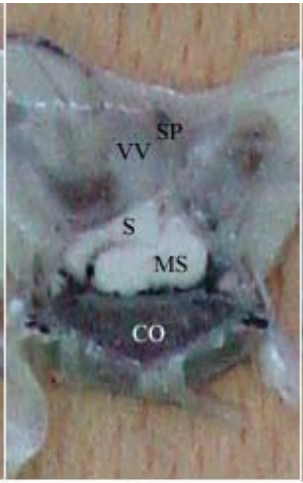

B

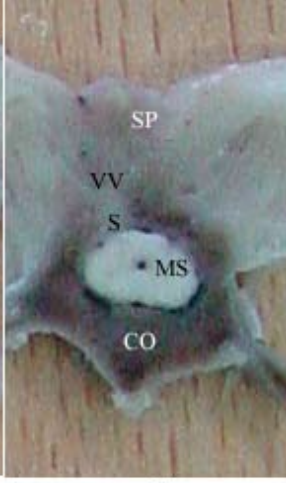

C
Fig.1. A. Sectional surface of unoperated rabbit's spinal column and medulla spinalis (SP: Spinous Process, MS: Medulla Spinalis, CO: Corpus)

B. Sectional surface of operated (hemilaminotomy) but unoperated rabbit's spinal column and medulla spinalis. This photo shows Grade III fibrosis formation (SP: Spinous Process, MS: Medulla Spinalis, CO Corpus, S: Scar, arrows shows hemilaminotomy opening).

C. Sectional surface of operated (hemilaminotomy) and treated (omega fatty acids solution) rabbit's spinal column and medulla spinalis. This photo shows Grade II fibrosis formation (SP: Spinous Process, MS: Medulla Spinalis, CO: Corpus, S: Scar, arrows shows hemilaminotomy opening)

The lumbosacral area was cleaned with providone iodine (Betadine, Adeka drug co. Samsun) soap and solution. The surgical area was draped with an aseptic transparent surgical drape to maintain aseptic condition. Around the surgical area was covered with a sterile cotton towels. Surgery was performed under the operating microscope (Zeiss Opmi, operating microscope).

A midline skin incision was made from L1 to L5. The lumbosacral fascia was cut bilaterally on the lateral edge of the spinous processes. The paraspinous muscles were subperiosteally dissected from the spinous process and laminae of L2 to L4. Self retaining retractor was used for retraction of the paraspinous muscles.

We previously described a new experimental model for examination peridural fibrosis. In this experiment we used hemilaminotomy procedure as described our previous study (Cokluk and Aydin, 2005). Hemilaminotomy was performed at L4 level. Hemilaminotomy defect, $2 \mathrm{~mm}$ in wide and $5 \mathrm{~mm}$ in long was made in left lamina with G4-
325D midas-rex diamond drill tip. Ligamentum flavum was removed with micro-scissor. Hemilaminotomy defect was also irrigated with sterile saline solution. 4-0 Vicryl sutures were used for suturing of the lumbosacral fascia. The skin was closed with 3-0 silk sutures.

In omega-3 group we put $250 \mathrm{mg}$ sterile omega-3 fatty acids solution (Marincap, $500 \mathrm{mg}$ liquid Omega-3 containing capsule, Kocak, Istanbul) after removing of ligamentum flavum. Four-0 Vicryl sutures were used for suturing of the lumbosacral fascia. The skin was closed

Table I. Grading of epidural scar tissue in according to gross inspection findings.

\begin{tabular}{|l|l|}
\hline Grade & $\begin{array}{l}\text { Gross inspection findings from coro- } \\
\text { nally sectioned specimens }\end{array}$ \\
\hline Grade I & $\begin{array}{l}\text { Scar tissue is located only in the para- } \\
\text { vertebral muscles }\end{array}$ \\
\hline Grade II & $\begin{array}{l}\text { Hemilaminotomy or laminectomy } \\
\text { defect was full-filled by scar tissue } \\
\text { without neuronal compression }\end{array}$ \\
\hline Grade III & $\begin{array}{l}\text { Hemilaminotomy or laminectomy de- } \\
\text { fect was full-filled by scar tissue with } \\
\text { neuronal compression }\end{array}$ \\
\hline
\end{tabular}

with 3-0 silk sutures.

The rabbits were transferred to its cages for recovery from anesthesia. One additional intramuscular injection of Penicillin (30 $000 \mathrm{U} / \mathrm{kg}$ ) was given approximately 24 hours postoperatively. The animals were allowed free access to food and water in their cages with a 12 hour light/dark cycle and a temperature of $22^{\circ} \mathrm{C}$. All the rabbits were fed a

Table II. Results of laminectomy and hemilaminotomy procedures.

\begin{tabular}{|l|lc|cc|}
\hline $\begin{array}{l}\text { Evaluation } \\
\text { Parameters }\end{array}$ & \multicolumn{4}{|c|}{ Groups } \\
\hline & \multicolumn{2}{|c|}{$\begin{array}{c}\text { Group I } \\
\text { (Hemilaminotomy) } \\
\mathrm{n}\end{array}$} & \multicolumn{2}{|c|}{$\begin{array}{c}\text { Group II } \\
\text { (Omega-3) } \\
\%\end{array}$} \\
\hline Grade I & - & - & 2 & 20 \\
\hline Grade II & 4 & 40 & 7 & 70 \\
\hline Grade III & 6 & 60 & 1 & 10 \\
\hline Mean score & $2,6 \pm 0,51$ & & \multicolumn{2}{|c|}{$1,9 \pm 0,56$} \\
\hline
\end{tabular}

standard rabbit diet after postoperative period.

Four weeks later from the operation, animals were sacrificed with a lethal dose of pentobarbital $(60 \mathrm{mg} / \mathrm{kg})$ administered via an ear vein. They were perfused and fixed with $10 \%$ neutral buffered formalin solution.

The lumbar spines were removed en-bloc from L1 through L5. The cranial portion was marked with 
a metallic marker. The specimen was immersed in $10 \%$ neutral buffered formalin for approximately 24 hours. Each specimen was then decalcified in 5\% formic acid for approximately 3 weeks.

Specimens were cut coronally for gross inspection. Two blocks of tissues were taken from each laminectomy site to be processed and embedded in paraffin. Permanent sections 6 to 8 microns thick were stained with hematoxylin and eosin.

Peridural scar tissue was graded according to our previous description in experimental hemilaminotomy model. According to this grading schema, peridural scar tissue on gross inspection in coronally sectioned specimens was graded into three grades as shoved in Table I. Grade I epidural scar tissue was scored with 1 point, Grade II with 2 point and Grade III with 3 point. Mann-Whitney-U test was used for statistical analysis.

\section{Results}

All animals were healty without any neurological deficit. There were no instances of superficial infection.

Group I (hemilaminotomy without omega-3 treatment): Six $(60 \%)$ hemilaminotomy without omega-3 sites were in Grade III fibrosis (Fig. 1 B). In these sites, fibrotic scar tissue penetrated into the hemilaminotomy defect and compressed the neural structures to the opposite direction. Remaining four (40\%) of hemilaminotomy sites were in Grade II fibrosis. In these levels, scar tissue penetrated into the hemilaminotomy site, adherent to the dural sac without neural compression. The average score of hemilaminotomy sites was $2.6 \pm 0.51$.

\section{Group II (omega-3 treatment):}

Seven $(70 \%)$ hemilaminotomy with omega-3 sites were in Grade II fibrosis (Fig. 1 C). In these rabbits hemilaminotomy defect was full-filled by scar tissue without neuronal compression. Two of them $(20 \%)$ were in Grade I fibrosis. In these rabbits scar tissue located only in the paravertebral muscles. Remaining $1(10 \%)$ of hemilaminotomy sites were in Grade III fibrosis. In these levels, scar tissue penetrated into the hemilaminotomy site, adherent to the dural sac without neural compression. The average score of hemilaminotomy with omega-3 treatment sites was 1,9 $\pm 0,56$. The results of hemilaminotomy without omega-3 (control) and hemilaminotomy with omega-3 (treatment group) were shown in Table II. Fig. 1 A, B, C show normal (unoperated), control (operated without treatment), and treated (omega-3) rabbit's spinal column and medulla spinalis.

The difference between these two groups was statistically significant $(\mathrm{p}<0.01)$.

\section{Discussion}

Epidural scar tissue formation is multifactorial in origin (Gabriel and Friedman, 1996; Dogulu et al., 2003). Many factors may affect this pathological process (Gabriel and
Friedman, 1996; Dogulu et al., 2003). The type of pathology, surgical technique selected for treatment the pathology, surgical instruments, gentle and minimally invasive techniques, personal tissue tendency for scar formation are some of factors. Surgically uncovering of dura mater and spinal roots from their biobarriers such as laminar bone and ligamentum flavum may be one of the main causes for peridural scar tissue formation and adhesions. These barriers separated them from the paravertebral soft tissues. After the operation, newly synthesized scar tissue can easily pass through the hemilaminotomy opening. The amount of newly synthesized scar tissue, the degree of neuronal compression, the severity of adhesions, advancement and invasion of scar tissue into the potential spaces are factors affecting clinical severity of pathology.

Many clinical and experimental researches has been conducted to prevent postoperative scar tissue formation and adhesions this pathological process still remains as a main cause of unsuccessful lumbar disc surgery (Robertson et al., 1993; Gabriel and Friedman, 1996; Dogulu et al., 2003).

Omega-3 fatty acids are polyunsaturated fatty acids classified as essential because they can not be synthesized in the body; they must be obtained from food. Important omega-3 fatty acids in human nutrition are alfa-linolenic acid, eicosapentaenoic acid, and docosahexaenoic acid.

During the surgical intervention, muscular, ligamentous and bone corridor are created for exposing and removing of disc material. This corridor can be created small or large in size according to the type of surgical intervention. A hemilaminatomy procedure with removing of a piece of laminar bone and ligamentum flavum is generally used for reach down to the affected disc level. After the operation, newly synthesized fibrotic tissue may produce new neurological symptoms and lead to the failed back surgery even in small hemilaminotomy openings (Gabriel and Friedman, 1996).

In recent studies in rabbit's laminectomy model, carbohydrate polymer was found to be effective to prevent peridural fibrosis (Robertson et al., 1993). A polyvinyl alcohol hydrojel membrane was also found to be effective as an anti-adhesive material in a feline postlaminectomy model (Robertson et al., 1993). Hyaluronic acid and sodium hyaluronate solution have yielded some promising results in animal models (Abitbol et al., 1994). Fat grafts have been found to decrease dural adhesions or scar density in animal models more effectively than surgical, Avitene, collagen, Gelfoam or polyglactin 910 (Nussbaum et al., 1990; Minamide et al., 1999). In our study, we used omega-3 fatty acids solution to prevent epidural fibrosis and peridural adhesions in our modified hemilaminotomy model in rabbits.

Our study demonstrated that omega-3 treatment group has promising results in the preventing of epidural fibrosis formation in comparison with control rabbits. In the hemilaminotomy group, the ratio of Grade III epidural fibrosis was found as $60 \%$. The meaning of Grade III 
fibrosis formation is the presence of epidural fibrosis inside the hemilaminotomy opening with neuronal compression inside the canal. The ratio of Grade I and Grade II fibrosis formation was found as $40 \%$ in this group of rabbits. In omega-3 treatment group, the ratio of Grade III epidural fibrosis was found as 10\%. Remaining $90 \%$ were Grade I and II fibrosis. In this group $70 \%$ of rabbits had Grade II fibrosis. In these rabbits epidural fibrosis fulfilled the hemilaminotomy opening without neuronal compression. In omega-3 group, $20 \%$ of rabbits had Grade I fibrosis. In these rabbits fibrosis formation was located in the paravertebral muscles over the hemilaminotomy openings. In the hemilaminotomy group (control group), there was no Grade I fibrosis formation.

This experimental study evaluated the success of omega-3 in the preventive effect of neuronal compression and adhesions by newly synthesized scar tissue in our rabbit hemilaminotomy model. Omega-3 solution seems to be an effective treatment modality in the prevention of peridural excessive scar tissue formation. For clinical application it is necessary to investigate further experimental studies.

\section{REFERENCES}

Abitbol, J.J., Lincoln T.L., Lind B.I., Amiel D., Akeson W.H., Garfin S.R., 1994. Preventing postlaminectomy adhesion: a new experimental model. Spine. 19, 1809-1814

Cokluk, C., Aydin, K., 2005. Experimental rabbit hemilaminotomy model in the evaluation of peridural fibrosis: A minimally invasive peridural fibrosis model. Minim. Invas. Neurosurg. 48, 235-239

Dogulu, F., Kurt, G., Emmez, H., Erdem, O., Memis, L., Baykaner, K., Ceviker, N., 2003. Topical mitomycin C-induced inhibition of postlaminectomy peridural fibrosis in rabbits. J. Neurosurg. 99, 76-79

Gabriel, E.M., Friedman, A.H., 1996. The failed back surgery syndrome. In: Wilkins RH, Rengachary DD, editors. Neurosurgery. McGraw-Hill, New York, 3863-3870

Minamide, A., Ilashizume, H., Yoshida, M., 1999. Effects of basic fibroblast growth factor on spontaneous resorption of hemiated intervertebral discs: an experimental study in the rabbit. Spine. 24, 940-945

Nussbaum, C.E., McDonald, J.V., Baggs, R.B., 1990. Use of Vicryl (polyglactin 910) mesh to limit epidural scar formation after laminectomy. Neurosurgery. 26, 649-654

Robertson, J.T., Meric, A.L., Dohan, F.C. Jr, Schweitzer, J.B., Wujek, J.R., Ahmad, S., 1993. The reduction of postlaminectomy peridural fibrosis in rabbits by a carbohydrate polymer. J. Neurosurg. 79, 89-95 